\title{
Using a Passage Retrieval System to Support Question Answering Process ${ }^{1}$
}

\author{
Fernando Llopis, José Luis Vicedo and Antonio Ferrández \\ Departamento de Lenguajes y Sistemas Informáticos \\ University of Alicante \\ Alicante, Spain \\ \{llopis, vicedo, antonio\}@dlsi.ua.es
}

\begin{abstract}
Previous works in Information Retrieval show that using pieces of text obtain better results than using the whole document as the basic unit to compare with the user's query. This kind of IR systems is usually called Passage Retrieval (PR). This paper discusses the use of our PR system in the question answering process (QA). Our main objective is to examine if a PR system provide a better support for QA than Information Retrieval systems based in the whole document..
\end{abstract}

\section{Introduction}

Given a user's query, Information Retrieval (IR) systems return a set of documents, which are sorted by the probability of containing the required information. Since IR systems return whole documents, there is an additional work for the user, who has to read the whole document to search for the required information. However, when searching for specific information, this last user's task can be carried out by Question Answering systems (QA), which are tools that usually work on the output of an IR system, and try to return the precise answer to the user's query.

IR systems work on measuring the similarity between each document and the query by means of several formulas that typically use the frequency of query terms in the documents. This way of measuring means that larger documents could have a greater chance at being considered relevant, because of the higher number of terms that could coincide with that of the query.

In order to solve this problem, some IR systems measure the similarity in accordance with the relevance of the pieces of adjoining text that form the documents, where these pieces of text are called passages. These kinds of IR systems, which are usually called

1 This paper has been partially supported by the Spanish Government (CICYT) project number TIC2000-0664-C02-02 
Passage Retrieval (PR), allows that the similarity measure is not affected by the size of the document. PR systems return the precise piece of text where it is supposed to find the answer to the query, a fact that is especially important when large documents are returned. PR systems are more complex than IR, since the number of textual units to compare is higher (each document is formed by several passages) and the number of tasks they accomplish is higher too (above all when passage splitting is performed after processing each query as it is proposed in [7]). Nevertheless, the complexity added to the system is rewarded with a significant increase in the performance of the system. For example, in [1] the improvement reaches a $20 \%$, and in [7] it does a $50 \%$.

As well as obtaining better precision than document retrieval systems, PR return the most relevant paragraphs to a query, information that could be used as input by a QA system. This fact allows reducing QA systems computational complexity (they work on small fragments of text instead of documents) and improves QA's precision, since the passage list retrieved is more relevant to the query than the results of a document retrieval system.

The PR system presented in this paper is called IR-n. It defines a novel passage selection model, which forms the passages from sentences in the document. IR-n has been used in the last Cross-Language Evaluation Forum (CLEF-2001) and in the last Text REtrieval Conference (TREC-2001) for the Question Answering track. Part of the better performance obtained in TREC-2001 with reference to TREC-2000, is due to IR-n.

The following section presents PR and QA backgrounds. Section 3 describes the architecture of our proposed PR system. In section 4, we give a detailed account of the tests, experiments and results obtained. Finally, we present the conclusions of this work.

\section{Backgrounds in Question Answering and Passage Retrieval}

The Computational Linguistic community has shown a recent interest in QA, and it comes after developing Information Extraction systems, which have been evaluated in Message Understanding Conferences (MUC). Specifically, the interest was shown when in TREC-8, there appears a new track on QA that tries to benefit from largescale evaluation, that was previously carried out on IR systems, in previous TREC conferences.

If a QA system wants to successfully obtain a user's request, it needs to understand both texts and questions to a minimum level. That is to say, it has to carry on many of the typical steps on natural language analysis: lexical, syntactical and semantic. This analysis takes much more time than a statistical analysis that is carried out in IR. Besides, as QA has to work with as much text as IR, and the user needs the answer in a limited period of time, it is usual that an IR system processes the query and after, the QA system will continue with its output. In this way, the time of analysis is greatly decreased. 
Some of the best QA systems are the following: [2],[3],[10],[5] After studying these systems, it seems agreeable the following general architecture, that is formed by four modules, where document retrieval module is accomplished by using IR technology:

- Question Analysis.

- Document Retrieval.

- Passage Selection.

- Answer Extraction.

Different IR models are used at the Document Retrieval stage in QA systems however, the best results are obtained by systems that apply PR techniques for this task.

The main differences between different PR systems are the way that they select the passages, that is to say, what they consider a passage and its size. According to the taxonomy proposed in [1], the following PR systems can be found: discourse-based model, semantic model and window model. The first one uses the structural properties of the documents, such as sentences or paragraphs (e.g. the one proposed in [9], [12]) in order to define the passages. The second one divides each document into semantic pieces, according to the different topics in the document (e.g. those in [4]). The last one uses windows of a fixed size (usually a number of terms) to form the passages [1], [6].

It would seem coherent that discourse-based models are more effective since they use the structure of the document itself. However, the problem with them is that the results could depend on the writing style of the document's author. On the other hand, window models have the advantage that they are simpler to use, since the passages have a previously known size, whereas the remaining models have to bear in mind the variable size of each passage. Nevertheless, discourse-based and semantic models have the advantage that they return logical and coherent fragments of the document, which is important if these IR systems are used for other applications such as Question Answering.

The passage extraction model that we are proposing allows us to benefit from the advantages of discourse-based models since logical information units of the text, such as sentences, form the passages. Moreover, another novel proposal in our PR system is the relevance measure, which unlike other discourse-based models, is not calculated from the number of passage terms, but from the fixed number of passage sentences. This fact, allows a simpler calculation of this measure unlike other discourse-based or semantic models. Although we are using a fixed number of sentences for each passage, we consider that our proposal differs from the window models since our passages do not have a fixed size (i.e. a fixed number of words) because we are using variable length sentences. 


\section{System Overview}

In this section, we describe the architecture of the proposed PR system, namely IR-n, focusing on its two main modules: the indexation and the document extraction modules.

\subsection{Indexation Module}

The main aim of this module is to generate the dictionaries that contain all the required information for the document-extraction module. It requires the following information for each term:

- The number of documents that contain the term.

- For each document:

- The number of times that the term appears in the document.

- The position of each term in the document: the number of sentence and position in the sentence.

Where we consider as terms, the stems produced by the "Porter stemmer" on those words that do not appear in a list of stop-words, list that is similar to those used in IR systems. For the query, the terms are also extracted in the same way, that is to say, their stems and positions in the query one query word that do not appear in the list of stop-words.

\subsection{Document Extraction Module}

This module extracts the documents according to it's similarity with the user's query. The scheme in this process is as follows:

1. Query terms are sorted according to the number of documents in which they appear, where the terms that appear in fewer documents are processed firstly.

2. The documents that contain some query term are extracted.

3. The following similarity measure is calculated for each passage $p$ with the query $q$ :

Similarity_measure $(\mathrm{p}, \mathrm{q})=\sum_{t \in p \wedge q} \mathrm{~W}_{\mathrm{p}, \mathrm{t}} * \mathrm{~W}_{\mathrm{q}, \mathrm{t}}$

Where:

$W_{p, t}=\log _{\mathrm{e}}\left(\mathrm{f}_{\mathrm{p}, \mathrm{t}}+1\right)$.

$f_{p, t}$ is the number of times that the term $t$ appears in the passage $p$.

$W_{q, t}=\log _{\mathrm{e}}\left(\mathrm{f}_{\mathrm{q}, \mathrm{t}}+1\right) *$ idf.

$f_{q, t}$ is the number of times that the term $t$ appears in the query $q$.

$i d f=\log _{\mathrm{e}}\left(\mathrm{N} / \mathrm{f}_{\mathrm{t}}+1\right)$.

$N$ is the number of documents in the collection.

$f_{t}$ is the number of documents that contain the term $t$. 
4. Each document is assigned the highest similarity measure from its passages.

5. The documents are sorted by their similarity measure.

6. The documents are presented according to their similarity measure.

As it will be noted, the similarity measure is similar to the cosine measure presented in [11]. The only difference is that the size of each passage (the number of terms) is not used to normalise the results. This difference makes the calculation simpler than other discourse-based PR systems or IR systems, since the normalization is accomplished according to a fixed number of sentences per passage. Another important detail to notice is that we are using $N$ as the number of documents in the collection, instead of the number of passages. That is because in [7] it is not considered relevant for the final results.

The optimum number of sentences to consider per passage is experimentally obtained. It can depend on the genre of the documents, or even on the type of the query as it is suggested in [6]. We have experimentally considered a fixed number of 20 sentences for the collection of documents with which we worked [8]. Table 1 presents the experiment where the 20 sentences per passage obtained the best results.

Table 1. Precision results obtained on Los Angeles Times collection with different number of sentences per passage.

\begin{tabular}{|c|c|c|c|c|c|c|}
\hline & \multicolumn{7}{|c|}{ Precision IR-n } \\
\hline Recall & 5 Sent. & 10 Sent. & 15 Sent. & 20 Sent. & 25 Sent. & 30 Sent. \\
\hline 0.00 & 0.6378 & 0.6508 & 0.6950 & 0.7343 & 0.6759 & 0.6823 \\
\hline 0.10 & 0.5253 & 0.5490 & 0.5441 & 0.5516 & 0.5287 & 0.5269 \\
\hline 0.20 & 0.4204 & 0.4583 & 0.4696 & 0.4891 & 0.4566 & 0.4431 \\
\hline 0.30 & 0.3372 & 0.3694 & 0.3848 & 0.3964 & 0.3522 & 0.3591 \\
\hline 0.40 & 0.2751 & 0.3017 & 0.2992 & 0.2970 & 0.2766 & 0.2827 \\
\hline 0.50 & 0.2564 & 0.2837 & 0.2678 & 0.2633 & 0.2466 & 0.2515 \\
\hline 0.60 & 0.1836 & 0.1934 & 0.1809 & 0.1880 & 0.1949 & 0.1882 \\
\hline 0.70 & 0.1496 & 0.1597 & 0.1517 & 0.1498 & 0.1517 & 0.1517 \\
\hline 0.80 & 0.1213 & 0.1201 & 0.1218 & 0.1254 & 0.1229 & 0.1279 \\
\hline 0.90 & 0.0844 & 0.0878 & 0.0909 & 0.0880 & 0.0874 & 0.0904 \\
\hline 1.00 & 0.0728 & 0.0722 & 0.0785 & 0.0755 & 0.0721 & 0.0711 \\
\hline
\end{tabular}


Table 2. Experiments with a different number of overlapping sentences

\begin{tabular}{|c|c|c|c|}
\hline Recall & $\begin{array}{c}\text { IR-n with } \\
\text { 1 overlap. }\end{array}$ & $\begin{array}{c}\text { IR-n with } \\
\text { 5 overlap. }\end{array}$ & $\begin{array}{c}\text { IR-n 10 } \\
\text { overlap. }\end{array}$ \\
\hline 0.00 & 0.7729 & 0.7211 & 0.7244 \\
\hline 0.10 & 0.7299 & 0.6707 & 0.6541 \\
\hline 0.20 & 0.6770 & 0.6072 & 0.6143 \\
\hline 0.30 & 0.5835 & 0.5173 & 0.5225 \\
\hline 0.40 & 0.4832 & 0.4144 & 0.4215 \\
\hline 0.50 & 0.4284 & 0.3704 & 0.3758 \\
\hline 0.60 & 0.3115 & 0.2743 & 0.2759 \\
\hline 0.70 & 0.2546 & 0.2252 & 0.2240 \\
\hline 0.80 & 0.2176 & 0.1914 & 0.1918 \\
\hline 0.90 & 0.1748 & 0.1504 & 0.1485 \\
\hline 1.00 & 0.1046 & 0.0890 & 0.0886 \\
\hline Medium & 0.4150 & 0.3635 & 0.3648 \\
\hline
\end{tabular}

As said, the proposed PR system can be classified into discourse-based models since it is using variable-sized passages that are based on a fixed number of sentences (but with a different number of terms per passage). The passages overlap each other, that is to say, let us suppose that the size of the passage is $N$ sentences, then the first passage will be formed by the sentences from 1 to $\mathrm{N}$, the second one from 2 to $\mathrm{N}+1$, and so on. We have decided to overlap just one sentence based on the following experiment, where several numbers of overlapping sentences have been tested. In this experiment, Table 2, it will observed that only one overlapping sentence obtained the best results.

\section{Evaluation}

This section presents the experiment proposed for evaluating our approach and the results obtained. The experiment has been run on the TREC-9 QA Track question set and document collection.

\subsection{Data Collection}

TREC-9 question test set is made up by 682 questions with answers included in the document collection. The document set consists of 978,952 documents from the TIPSTER and TREC following collections: AP Newswire, Wall Street Journal, San Jose Mercury News, Financial Times, Los Angeles Times, Foreign Broadcast Information Service. 


\subsection{Experiment}

In order to evaluate our proposal we decided to compare the quality of the information retrieved by our system with the ranked list retrieved by the ATT information retrieval system.

First, ATT IR system was used for retrieving the first 1000 relevant documents for each question. Second, our approach was applied over these 1000 retrieved documents in order to re-rank the list obtained by ATT system for each question. This process was repeated for different passage lengths. These lengths were of 5, 10, 15, 20, 25 and 30 sentences.

The final step was to compare the list retrieved by ATT system and the four lists obtained by our system. Table 3 shows the precision obtained in each test. The precision measure is defined as the number of answers included at 5, 10, 20, 30, 40, 50, 100 and 200 first documents or paragraphs retrieved in each list.

\subsection{Results Obtained}

Table 3 shows the number of correct answers included into the top $\mathrm{N}$ documents of each list for the 681 test questions. The first column indicates the number $\mathrm{N}$ of first ranked documents selected for measuring the precision of each list. The second column shows the results obtained by ATT list. The remaining columns show the precision obtained by our system for passages of 5, 10, 15, 20, 25 an 30 sentences.

It can be observed that our proposal obtains better results than the original model, being 20 sentences the optimum passage length for this task.

Table 3. Obtained results on TREC collection and 681 TREC queries.

\begin{tabular}{|c|c|c|c|c|c|c|c|}
\hline & & \multicolumn{5}{|c|}{ IR-n system } \\
\hline $\begin{array}{c}\text { Answer } \\
\text { included }\end{array}$ & $\begin{array}{c}\text { ATT } \\
\text { system }\end{array}$ & 5 Sent. & 10 Sent. & 15 Sent. & 20 Sent. & 25 Sent & 30 Sent. \\
\hline At 5 docs & 442 & 444 & 464 & 488 & 508 & 532 & 531 \\
\hline At 10 docs & 479 & 505 & 531 & 549 & 561 & 570 & 572 \\
\hline At 20 docs & 517 & 551 & 573 & 584 & 595 & 599 & 599 \\
\hline At 30 docs & 539 & 575 & 596 & 600 & 612 & 617 & 618 \\
\hline At 50 docs & 570 & 599 & 611 & 623 & 624 & 637 & 637 \\
\hline At 100 docs & 595 & 614 & 631 & 640 & 644 & 650 & 646 \\
\hline At 200 docs & 613 & 634 & 643 & 648 & 654 & 655 & 655 \\
\hline
\end{tabular}




\section{Conclusions and Future Works}

In this paper, a passage retrieval system for QA purposes has been presented. This model can be included in the discourse-based models since it is using the sentences as the logical unit to divide the document into passages. The passages are formed by a fixed number of sentences. As results show, this approach performs better than a good IR system for QA tasks. Results seem better if we take into account that our passage approach returns only a small piece of the whole document. This fact enhances our results because the amount of text that a QA system has to deal with is much smaller than using a whole document. This way, our approach achieves a reduction in QA processing time without affecting system performance.

Several areas of future work have appeared while analysing results. First, we have to investigate the application of question expansion techniques that allow increasing system precision. Second, we have to measure the performance of our system by applying directly our paragraph retrieval method over the whole document collection instead of using it for reordering a given list. And thirdly, it would be very useful for QA systems to develop a passage validation measure to estimate the minimum number of passages that a QA system has to process to be sure that it contains the answer to the question. All these strategies need to be investigated and tested.

\section{References}

[1] Callan, J. Passage-Level Evidence in Document Retrieval. In Proceedings of the 17 th Annual ACM SIGIR Conference on Research and Development in Information Retrieval, Dublin, Ireland, 1994, pp. 302-310.

[2] Clarke, C.; Derek, C.; Kisman, I. and Lynam, T. R. Question Answering by Passage Selection (MultiText Experiments for TREC9). In Nineth Text REtrieval Conference, 2000 .

[3] Harabagiu, S.; Moldovan, D.; Pasca, M.; Mihalcea, R.; Surdeanu, M.; Bunescu, R.; Gîrju, R.; Rus, V. and Morarescu, P. FALCON: Boosting Knowledge for Answer Engines. In Nineth Text REtrieval Conference, 2000.

[4] Hearst, M. and Plaunt, C. Subtopic structuring for full-length document access. Proceedings of the Sixteenth Annual International ACM SIGIR Conference on Research and Development in Information Retrieval, June 1993, Pittsburgh, PA, pp 59-68

[5] Ittycheriah, A.; Franz, M.; Zu, W. and Ratnaparkhi, A. IBM's Statistical Question Answering System. In Nineth Text REtrieval Conference, 2000.

[6] Kaskiel, M. and Zobel, J. Passage Retrieval Revisited SIGIR '97: Proceedings of the 20th Annual International ACM July, 1997, Philadelphia, PA, USA, pp 27-31

[7] KaszKiel, M. and Zobel, J. Effective Ranking with Arbitrary Passages. Journal of the American Society for Information Science, Vol 52, No. 4, February 2001, pp 344-364.

[8] Llopis, F. and Vicedo, J. Ir-n system, a passage retrieval system at CLEF 2001 Working Notes for the Clef 2001 Darmstdt, Germany, pp 115-120

[9] Namba, I Fujitsu Laboratories TREC9 Report. Proceedings of the Tenth Text REtrieval Conference, TREC-10. Gaithersburg,USA. November 2001, pp 203-208 
[10] Prager, J.; Brown, E.; Radev, D. and Czuba, K. One Search Engine or Two for QuestionAnswering. In Nineth Text REtrieval Conference, 2000.

[11] Salton G. Automatic Text Processing: The Transformation, Analysis, and Retrieval of Information by Computer, Addison Wesley Publishing, New York. 1989

[12] Salton, G.; Allan, J. Buckley Approaches to passage retrieval in full text information systems. In R Korfhage, E Rasmussen \& P Willet (Eds.) Prodeedings of the 16 th annual international ACM-SIGIR conference on research and development in information retrieval. Pittsburgh PA, pp 49-58 